\title{
A Mathematical Optimization Model To Support Decision Making For Fast Food Enterprises
}

Jess S. Boronico, University of New Haven

Alexandros Panayides, (E-mail: panayidesa@wpunj.edu), William Paterson University

Lori Goldstein, (E-mail: lgoldstein@comcast.net)

\begin{abstract}
This paper investigates the determination of the optimum time to begin shut down procedures for fast food establishments. The model developed in the paper provides conditions for the number of minutes prior to closing time to begin shut down procedures and the optimal number of items to have prepared together with a comparative static analysis. Illustrative results are presented based on empirical data obtained from a pretzel establishment study.
\end{abstract}

\section{INTRODUCTION AND LITERATURE REVIEW}

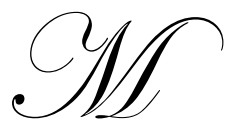

uch work has been devoted to the application of quantitative methods to the fast food industry (Ball, 1992). Topics discussed include inventory methods, process analysis, productivity evaluations, employment trends, and marketing research, with much of this research focused on competitive advantage in an open market. In response to current changes in these markets, many industries, including the fast food industry, have witnessed a significant trend towards the substitution of technology for labor. This substitution is expected to result in significant economies of scale through which additional competitive advantage may be obtained. Other service industries have also been faced with the problem of understanding how technology can be applied to increase efficiency and lower production costs, such as the Postal Service (Crew and Kleindorfer, 1992).

This paper examines an interesting problem related to the use of technology, and faced by many fast food providers: the determination of the appropriate time to begin shut down procedures. Shutting down the grill/oven is ordinarily the catalyst that initiates the entire shut down procedure. If the process can be conducted in a more efficient manner, it is anticipated that the establishment will realize some level of cost savings, as shown in a subsequent section. Dialog with personnel from fast food franchises suggests that the problem of shut down time is ordinarily not considered by fast food operators, whose management contends that grills usually remain open until the establishment closes. Therefore, the determination of optimal shut down policies may be of interest to the management of these operations. This paper focuses on one such operation: a particular pretzel fast food establishment located in a shopping mall.

As mentioned above, much of the current fast food establishment's literature focuses on competitive advantage. For example, Yasin and Yavas (1992) develop a specific inventory sampling and control system for a fast food operation in Atlanta. Donthu and Yoo (1998) use Data Envelopment Analysis (DEA) to track the relative productivity of twenty-four fast food restaurants. Farsad and LeBruto (1993) apply optimal reorder strategies subject to varying average daily demand. Gavish and Graves (1980) consider the problem of evaluating when a production facility should be active or idle, and develop an algorithm to minimize the expected cost of one product production process. Their research is based on preliminary results established by Sobel (1968), who investigates the general problem of start up and shut down policies for production processes. Despite the wealth of literature noted above, shut down procedures, per se, have not been specifically addressed in the fast food literature. The model developed in this paper bears the familiar earmarks of some standard optimization problems' characteristics, such as those obtained from the classical newsboy inventory problem. These somewhat familiar characteristics are embodied within the 
particular solutions derived for the grill-closing model developed here, which addresses both an optimal stocking policy (modeled with a single perishable item) in addition to an optimal timing policy.

Concerning the newsboy problem, a vast literature exists. For example, an excellent summary of many of the significant contributions made in this area can be found in Khouja, Mehrez, and Rabinowitz (1996). Some of the more recent applications of the newsboy problem and its extensions include Khouja (1996), who investigates an emergency supply option for out of stock books, Lau and Lau (1997), who consider midseason replenishment policies in the garment industry, and Lippman and McCardle (1994), who evaluate demand split among several competitive firms. Nahmais (1993) considers the expected costs for a typical newsboy model with stochastic demand. His results are similar to those indicated in Freis (1975) as well as older studies such as the seminal studies conducted by Arrow (1958). Lau and Lau (1997) consider an extension to the Nahmias study: their work is applicable to retailers of a variety of seasonal items.

The model developed in this paper is based on conversations with managers and employees of small fast food establishments. The model highlights the joint utilization of current desktop technology with quantitative modeling for small establishments in the fast food industry. Desktop technology can often be used as a viable substitute when analytical solutions are intractable; however, management is often unaware that this technology can improve the resulting recommendations of their decision-making. The integrated approach of using technology and quantitative modeling has become increasingly popular with larger fast food establishments such as Burger King (Swart and Donno 1981). Unfortunately, smaller establishments sometimes lack either the resources or the expertise to initiate an integrated approach; hence, the model presented in this paper provides particular implications for these smaller establishments.

The remainder of the paper is organized as follows. The theoretical model is developed in section two, where optimal solutions are characterized. These solutions are then applied to a pretzel establishment study in section three. Comparative statics and sensitivity analysis are pursued in section four, conclusions in section five and implications for future research in section six.

\section{THE MODEL}

A problem that all fast food managers contend with involves the timing of shut down procedures as closing time approaches: this involves determining the number of minutes prior to closing time to begin these procedures $(t)$ and the number of items $(x)$ to have prepared at time $t$. Let $n$ represent the number of arrivals to the fast food establishment stand over $t$ and designate $P_{n}(t)$ the probability of $n$ arrivals over time $t$. We assume that arrivals follow a Poisson Process. That is,

$P_{n}(t)=e^{-\lambda t} \frac{(\lambda t)^{n}}{n !}, \quad n=0,1,2, \ldots$

Let $\lambda$ denote the average arrival rate: we assume that $\lambda$ is constant. Further, $c$ represents the hourly wage for an employee, $g$ is the time required to close/shut down the grill, $b$ represents the wholesale cost for an item, $s$ denotes the selling price for an item, and $l$ is the lost sales cost per item (which may also be written as $s-b$ ).

Given parameters $\lambda, c, b$, and $s$, the objective is to determine the value of the $(x, t)$ pair that minimizes the following cost function:

$$
C(x, t)=\operatorname{Max}\left[0, \frac{c}{60}(g-t)\right]+b \sum_{n=0}^{x}(x-n) P_{n}(t)+l \sum_{n=x+1}^{\infty}(n-x) P_{n}(t)
$$


where $\operatorname{Max}\left[0, \frac{c}{60}(g-t)\right]$ represents the wage cost, $b \sum_{n=0}^{x}(x-n) P_{n}(t)$ represents the leftover items cost, i.e., the over preparing cost, $l \sum_{n=x+1}^{\infty}(n-x) P_{n}(t)$ represents the lost sales cost, i.e., the under preparing cost, and $P_{n}(t)$ is the Poisson probability function given above. Utilizing substitution and algebraic manipulation, it is possible to rewrite the objective (2) as follows:

$$
C(x, t)=\operatorname{Max}\left[0, \frac{c}{60}(g-t)\right]+x(b+l) \sum_{n=0}^{x} P_{n}(t)-(b+l) \sum_{n=0}^{x} n P_{n}(t)+l(\lambda t-x)
$$

We now consider the determination of the optimal vector $\left(x^{*}, t *\right)$. To begin, for any feasible value of $t \in[0, \infty)$ the optimal solution for $x$ adheres to the following prescription:

Decision Rule: If the cost of preparing one more item is less than the average cost of preparing one item, then prepare one more item.

In mathematical terms: If $C(x+1, t)<C(x, t)$ then prepare one more item.

More specifically:

Theorem 1: (a) Prepare one more item as long as:

$$
\sum_{n=0}^{x-1} P_{n}(t)=\frac{l-\frac{c}{\lambda}}{b+l} \quad \text { for } \quad \mathrm{t}<\mathrm{g}
$$

or

$$
\sum_{n=0}^{x-1} P_{n}(t)=\frac{l}{b+l} \quad \text { for } \quad \mathrm{t}>\mathrm{g}
$$

(b) For any time t, the minimum cost occurs at that positive integer $\mathrm{x}$ for which

$$
\sum_{n=0}^{x-1} P_{n}(t)<\frac{l}{b+l} \leq \sum_{n=0}^{x} P_{n}(t)
$$

Proof: See Appendix.

The model's solution (5)-(7) has similarities to both the newsboy problem and the lost sales EOQ model. In general, the number of items to prepare is a function of the demand probabilities $P_{n}(t)$ and the over $(b)$ and underpreparing $(l)$ costs. Regarding the time to close the grill, numerical results suggest that the optimal shut down time is either $t=(0, g)$, that is, it appears that either the grill does not get shut down until closing time, or else gets shut down so that the grill cleaning is completed at shut down time. The former corresponds to the lost sales case where no sales should be last, whereas the latter corresponds to the case where if any sales are lost (i.e., by closing the grill early), then the grill should be closed as early as possible with all potential sales lost. Note that it is intuitive to recognize that $t$ is bound over the interval $[0, g]$.

We do not propose a mathematical closed form nature of the solution vector, but utilize an iterative approach as demonstrated in the following section. 


\section{EMPIRICAL RESULTS}

To test the model, data were collected from Penn Pretzel, a fast food establishment in a local shopping mall. Penn Pretzel sells pretzels with a variety of toppings, such as salt or cinnamon sugar. The establishment also sells a wide variety of beverages. The store manager usually puts the last batch of pretzels into the oven approximately 45 minutes before closing. The pretzels take 6-8 minutes to bake. Once the last batch of pretzels goes into the oven, the employees begin the shut down procedures. The owner of the establishment was interested in minimizing the employee costs as well as decreasing the number of pretzels that would have to be thrown away at the end of the evening. The arrivals to the establishment were monitored from the time the shut down process was begun until the establishment was closed for the evening. Data were collected for a weekend and a weekday day.

For one particular weekend day, the following data was compiled: $c=\$ 19.50, s=\$ 1.60, b=\$ .10, l=s-b=$ $\$ 1.50, \lambda=0.49$, and $g=52$ minutes to clean the grill. Table 1 illustrates the determination of the optimum $t$ and the corresponding costs (in dollars) for $x=0,1,2, \ldots, 60$. Note that for each $t$, Theorem 1 was utilized in obtaining the optimum value of $x$.

Table 1: Empirical Results - Weekend

\begin{tabular}{|c|c|c|}
\hline$\underline{\mathbf{t}}$ & $\underline{\mathbf{x}}$ & Total Cost \\
\hline 0 & 0 & 16.900 \\
\hline 1 & 2 & 16.750 \\
\hline 2 & 3 & 16.490 \\
\hline 3 & 3 & 16.210 \\
\hline 4 & 4 & 15.920 \\
\hline 5 & 5 & 15.620 \\
\hline 6 & 6 & 15.330 \\
\hline 7 & 6 & 15.040 \\
\hline 8 & 7 & 14.730 \\
\hline 9 & 8 & 14.430 \\
\hline 10 & 8 & 14.130 \\
\hline 11 & 9 & 13.820 \\
\hline 12 & 10 & 13.520 \\
\hline 13 & 10 & 13.220 \\
\hline 14 & 11 & 12.910 \\
\hline 15 & 12 & 12.600 \\
\hline 16 & 12 & 12.300 \\
\hline 17 & 13 & 11.990 \\
\hline 18 & 14 & 11.680 \\
\hline 19 & 14 & 11.370 \\
\hline 20 & 15 & 11.060 \\
\hline 21 & 15 & 10.750 \\
\hline 22 & 16 & 10.440 \\
\hline 23 & 17 & 10.130 \\
\hline 24 & 17 & 9.820 \\
\hline 25 & 18 & 9.510 \\
\hline 26 & 18 & 9.200 \\
\hline 27 & 19 & 8.880 \\
\hline 28 & 20 & 8.580 \\
\hline 29 & 20 & 8.260 \\
\hline 30 & 21 & 7.950 \\
\hline
\end{tabular}

\section{$\underline{\mathbf{t}}$}

31

32

33

34

35

36

37

38

39

40

41

42

43

44

45

46

47

48

49

50

51

52

53

54

55

56

57

58

59

60
Total Cost

7.640

7.320

7.020

6.700

6.390

6.070

5.760

5.450

5.130

4.820

4.500

4.190

3.880

3.560

3.250

2.930

2.620

2.300

1.990

1.670

1.350

1.040

1.050

1.060

1.070

1.080

1.090

1.100

1.110

1.120 
From Table 1 we can see that the cost is minimized when the grill is turned off 52 minutes before closing time (which is also the time required to clean the grill, $g$ ) with $x^{*}=33$ pretzels prepared when the grill is shut off at this time. As an aside, note that $\sum_{n=0}^{32} P_{n}(52)=0.9138<\frac{l}{b+l}=0.9375$ and $\sum_{n=0}^{33} P_{n}(52)=0.9389>0.9375$, which is consistent with Theorem 1.

We now consider a second empirical analysis, based on a weekday observation, where the following data applies: $c=\$ 13.00, s=\$ 1.60, b=\$ .10, l=s-b=\$ 1.50, \lambda=0.066$, and $g=55$ minutes to clean the grill. Table 2 , in similar fashion to Table 1 , provides the resulting optimum recommendation:

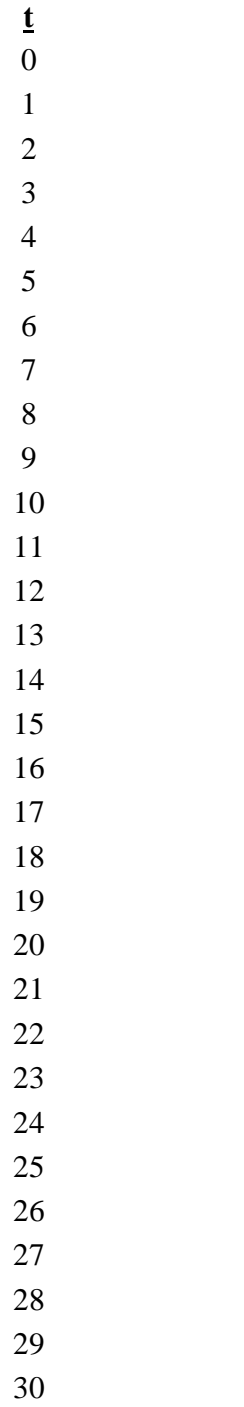

Table 2: Empirical Results - Weekday

$\underline{\text { Total Cost }} \underline{\text { t }}$

11.920

$11.800 \quad 32$

$11.580 \quad 33$

$11.380 \quad 34$

$11.180 \quad 35$

$10.980 \quad 36$

$10.790 \quad 37$

10.660

10.360

10.150

10.000

9.730

9.530

9.330

9.120

8.900

8.690

8.480

8.270

8.060

7.850

7.640

7.430

7.220

7.000

6.790

6.580

6.370

6.150

5.940

5.730

\begin{tabular}{c} 
Total Cost \\
\hline 5.530 \\
5.320 \\
5.100 \\
4.890 \\
4.670 \\
4.460 \\
4.250 \\
4.030 \\
3.820 \\
3.610 \\
3.400 \\
3.190 \\
2.980 \\
2.760 \\
2.550 \\
2.330 \\
2.120 \\
1.910 \\
1.690 \\
1.480 \\
1.270 \\
1.060 \\
0.850 \\
0.630 \\
0.417 \\
0.419 \\
0.422 \\
0.424 \\
0.428 \\
0.430 \\
\end{tabular}

$4 \quad 5.730$


From Table 2 we can see that the cost is minimized when the grill is turned off 55 minutes before closing time and with $x^{*}=7$ pretzels prepared when the grill is shut off. (Note again that $\sum_{n=0}^{6} P_{n}(55)=0.9242<\frac{l}{b+l}=0.9375$ and $\left.\sum_{n=0}^{7} P_{n}(55)=0.9679>0.9375\right)$

\section{COMPARATIVE STATICS AND SENSITIVITY ANALYSIS}

This section considers the sensitivity of the optimal solution with respect to changes in the problem's exogenous variables. In particular, we utilize the model to predict how the optimal values for the endogenous variables will respond to changes in some of the model's exogenous variables (wholesale pretzel cost, pretzel demand, time required to complete shut down procedures, and number of employees working at the end of the evening).

The wholesale cost of a pretzel is a parameter that will impact the shut down procedures. Each portion of pretzel dough has a wholesale cost of $\$ 4.00$. An experienced pretzel roller can make 50 pretzels from a batch of dough, an average roller can make 40, and a novice roller can make only 30 . As shown in table 2 , the experience of the pretzel employee does not affect the optimum $t$, which remains at 52 where $t=g$. However, the optimum $x$ and total cost values do fluctuate based on the experience level. Table 3 illustrates numerically these results as well the changes in the marginal cost. Note from the results that the marginal cost at the optimal solution decreases as the employee becomes increasingly proficient at rolling pretzels.

\begin{tabular}{c} 
Pretzels/Batch \\
\hline 49 \\
46 \\
43 \\
40 \\
37 \\
34 \\
31
\end{tabular}

\begin{tabular}{ccccc}
\multicolumn{4}{c}{ Table 3: Sensitivity Of Optimal Solution To Pretzel Cost $(\mathbf{B})$} & \\
$\underline{\mathbf{b}}$ & $\underline{\mathbf{t}^{*}}$ & $\frac{\mathbf{x}^{*}}{34}$ & $\frac{\text { Total Cost }}{\text { Marginal Cost }}$ \\
0.08163 & 52 & 34 & 0.886 & \\
0.08696 & 52 & 34 & 0.932 & 0.046 \\
0.09302 & 52 & 33 & 0.984 & 0.052 \\
0.10000 & 52 & 33 & 1.042 & 0.058 \\
0.10810 & 52 & 33 & 1.105 & 0.063 \\
0.11765 & 52 & 33 & 1.178 & 0.073 \\
0.12903 & 52 & & 1.266 & 0.088
\end{tabular}

Changes in the pretzel demand $\lambda$ will also impact on the optimum value for $x$ as well as total cost. As with the wholesale cost sensitivity analysis, $t^{*}=52$ (where $t=g$ ). For a typical weekday night, the demand is 0.07 per minute, as compared to a weekend night where demand is 0.49 . Based on the large range of optimum $x$ values between these two $\lambda$ values (see Table 4) it is important for the owner to estimate the number of pretzels to have on hand accurately. Each 0.05 increase in $\lambda$ represents a 2.6 unit increase in arrivals per minute. Each additional 2.6 customers that arrive per minute increase the optimum number of pretzels to have prepared by either three or four. Table 4 illustrates these results.

Next, we examine how changes in the time required to complete shut down procedures $(g)$ will affect the optimum $x$ and total cost. As before, in this sensitivity analysis the optimum $t$ equals $g$ (in general, this result has not been proven to hold for all realizations of parameter values). The changes in $g$, holding all other parameter values constant, are illustrated in Table 5. As expected, the quicker the establishment can be shut down, the smaller the resulting costs will be. It would be to the owner's advantage to strive to reduce the shut down time. 
Table 4: Sensitivity Of Optimal Solution To Demand Rate $(\lambda)$

$\begin{array}{llcc}\underline{\boldsymbol{\lambda}} & \frac{\mathbf{t}^{*}}{52} & \frac{\mathbf{x}^{*}}{5} & \text { Total Cost } \\ 0.05 & 52 & 9 & 0.36 \\ 0.10 & 52 & 12 & 0.49 \\ 0.15 & 52 & 16 & 0.59 \\ 0.20 & 52 & 19 & 0.68 \\ 0.25 & 52 & 22 & 0.76 \\ 0.30 & 52 & 25 & 0.82 \\ 0.35 & 52 & 28 & 0.88 \\ 0.40 & 52 & 31 & 0.94 \\ 0.45 & 52 & 34 & 1.00 \\ 0.50 & 52 & 37 & 1.05 \\ 0.55 & 52 & & 1.10\end{array}$

Table 5: Sensitivity Of Optimal Solution To Time Required To Complete Shutdown Procedures (G)

$\begin{array}{llll}\mathbf{g} & \frac{\mathbf{t}^{*}}{15} & \frac{\mathbf{x}^{*}}{12} & \text { Total Cost } \\ 15 & 15 & 15 & 0.58 \\ 20 & 20 & 18 & 0.66 \\ 25 & 25 & 21 & 0.73 \\ 30 & 30 & 24 & 0.80 \\ 35 & 35 & 27 & 0.86 \\ 40 & 40 & 29 & 0.92 \\ 45 & 45 & 32 & 0.97 \\ 50 & 50 & 35 & 1.02 \\ 55 & 55 & 38 & 1.07 \\ 60 & 60 & & 1.11\end{array}$

Lastly, as the employee cost is more substantial than the pretzel cost, we investigate changes in the number of employees working at the end of the evening. In this scenario, the third employee leaves work when the shut down begins. The remaining two employees execute the shut down procedures. As a result the wage cost is reduced by $\$ 6.50$ (the hourly wage for the employee) to $\$ 13.00$. Based on the stuff reduction by one, it has been observed that $g$ will increase by five minutes at $g=57$. In this scenario the minimum total cost of $\$ 1.09$ is achieved at $t^{*}=57$ (where $t=g$ ). The additional pretzel cost of $\$ 0.05$ over the optimum cost in table 1 can be attributed to the demand for the additional five minutes. However, this extra expense is more than offset by the $\$ 6.50$ wage savings.

\section{CONCLUSION}

This paper has considered the determination of the optimum time to begin shut down procedures in a fast food establishment and the amount of items to have prepared at that time. Based on the empirical results of a pretzel study, the operation should begin the shut down procedures exactly $g$ minutes before closing. At that point the shut down procedures will be completed at closing time. In this scenario there are no additional wage costs for overtime. The optimum amount of pretzels to have on hand when shut down begins depends on the demand rate, whose value fluctuates depending on the day of the week.

Even though the optimal time to begin shut down procedures cannot be obtained via an analytical method, the model has practical implications. With the help of a computer the owner of the pretzel establishment may be able to minimize the daily total costs with efficient shut down procedures. 
Table 6: Sensitivity Of Optimal Solution To Number Of Employees

$\begin{array}{ccc}\frac{\mathbf{t}^{*}}{0} & \frac{\mathbf{x}^{*}}{0} & \frac{\text { Total Cost }}{12.35} \\ 5 & 5 & 11.61 \\ 10 & 8 & 10.67 \\ 15 & 12 & 9.68 \\ 20 & 15 & 8.68 \\ 25 & 18 & 7.67 \\ 30 & 21 & 6.65 \\ 35 & 24 & 5.63 \\ 40 & 27 & 4.60 \\ 45 & 29 & 3.57 \\ 50 & 32 & 2.54 \\ 55 & 35 & 1.50 \\ 57 & 36 & 1.09 \\ 60 & 38 & 1.11\end{array}$

\section{SUGGESTIONS FOR FUTURE RESEARCH}

The model can be expanded in several directions. An interesting extension for future research would be to consider the problem of perishability if the last batch is prepared too early. Another extension may be to include a multi-period problem with dynamic programming to investigate the demand over one complete workday.

\section{REFERENCES}

1. Arrow, K. J., S. Karlin, and H. Scarf. Studies in the Mathematical Theory of Inventory and Production. Stanford: Stanford University Press, 1958.

2. Azoury, K. S. Bayes Solution to Dynamic Inventory Model under Unknown Demand Distribution. Management Science, September 1995: 1150-1160

3. Ball, S. Fast Food Operations and Their Management. England: Cheltenham.

4. Crew, M. A. and P. R. Kleindorfer (eds). The Economics of Postal Service. Boston: Kluwer Academic Publishers.

5. Donthu, N. and B. Yoo. Retail Productivity Assessment Using Data Envelopment Analysis. Journal of Retailing, Spring 1998: 89-105.

6. Farsad, B. and S. LeBruto. A Measured Approach to Food-Inventory Management. Cornell Hotel and Restaurant Administration Quarterly, June 1993: 90.

7. Fries, B. Optimal Ordering Policy for a Perishable Commodity with Fixed Lifetime. Operations Research, January-February 1975: 46-61.

8. Gavish, B. and S. Graves. A One-Production/Inventory Problem under Continuous Review Policy. Operations Research, September-October 1980: 1228-1236.

9. Khouja, M. A Note on the Newsboy Problem with an Emergency Supply Option. Journal of Operational Research Society, 1996(47): 1530-1534.

10. Khouja, M., A. Mehrez, and G. Rabinowitz. A Two-Item Newsboy Problem with Substitutability. International Journal of Production Economics, July 1996: 267-275.

11. Lau, H. and A. Hing-Ling Lau. Some Results on Implementing a Multi-Item Multi-Constraint Single-Period Inventory Model. International Journal of Production Economics, January 1997: 121-128.

12. Lau, H. and A. Hing-Ling Lau. Reordering Strategies for a Newsboy-type Product. European Journal of Operational Research, 1997(103): 557-572.

13. Lippman, S. and K. McCaedel. The Competitive Newsboy. Operations Research, January-February 1997: 54-65.

14. Nahmias, S. Production and Operations Management. New York: Irwin, 1997. 
15. Nandakumar, P. and T. Morton. Near Myopic Heuristics for the Fixed-Life Perishability Problem. Management Science, December 1993: 1490-1498.

16. Sobel, M. Optimal Average-Cost Policy for a Queue with Start-Up and Shut-Down Costs. Operations Research, 1969(17): 145-162.

17. Swart, W. and L. Donno. Simulation Modeling Improves Operations, Planning, and Productivity of Fast Food Restaurants. Interfaces, December 1981: 35-47.

18. Yasin, M. and U. Yavas. Improving the Quality and Efficiency of Fast Food Operations. International Journal of Retail and Distribution Management, July 1992: 28.

\section{APPENDIX:}

Theorem 1: (a) Prepare one more item as long as:

$$
\sum_{n=0}^{x-1} P_{n}(t)=\frac{l-\frac{c}{\lambda}}{b+l} \text { for } \quad \mathrm{t}<\mathrm{g},
$$

or

$$
\sum_{n=0}^{x-1} P_{n}(t)=\frac{l}{b+l} \quad \text { for } \quad \mathrm{t}>\mathrm{g} .
$$

(b) For any time t, the minimum cost occurs at that positive integer $\mathrm{x}$ for which

$$
\sum_{n=0}^{x-1} P_{n}(t)<\frac{l}{b+l} \leq \sum_{n=0}^{x} P_{n}(t)
$$

Proof: (a) Recall the cost function

$$
C(x, t)=\operatorname{Max}\left[0, c_{0}(g-t)\right]+b \sum_{n=0}^{x}(x-n) P_{n}(t)+l \sum_{n=x+1}^{\infty}(n-x) P_{n}(t) .
$$

First, when $t>g$ follows that

$$
C(x, t)=b x P_{0}(t)+b \sum_{n=1}^{x}(x-n) P_{n}(t)+l \sum_{n=x+1}^{\infty}(n-x) P_{n}(t) .
$$

To find the optimal solution, differentiation of the above expression with respect to $t$ yields

$$
\begin{aligned}
& \frac{\partial C}{\partial t}=-b \lambda x P_{0}+b \lambda \sum_{n=1}^{x}(x-n)\left[P_{n-1}(t)-P_{n}(t)\right]+l \lambda \sum_{n=x+1}^{\infty}(n-x)\left[P_{n-1}(t)-P_{n}(t)\right] \\
& =-b \lambda \sum_{n=0}^{x}(x-n) P_{n}(t)+b \lambda \sum_{n=1}^{x}(x-n) P_{n-1}(t)-l \lambda \sum_{n=x+1}^{\infty}(n-x) P_{n}(t)+l \lambda \sum_{n=x+1}^{\infty}(n-x) P_{n-1}(t) \\
& =-b \lambda \sum_{n=0}^{x}(x-n) P_{n}(t)+b \lambda \sum_{m=1}^{x-1}(x-m-1) P_{m}(t)-l \lambda \sum_{n=x+1}^{\infty}(n-x) P_{n}(t)+l \lambda \sum_{m=x}^{\infty}(m+1-x) P_{m}(t) \\
& =-b \lambda \sum_{m=0}^{x-1} P_{m}(t)+l \lambda \sum_{m=x}^{\infty} P_{m}(t)=\lambda\left[-b \sum_{m=0}^{x-1} P_{m}(t)+l\left(1-\sum_{m=0}^{x-1} P_{m}(t)\right)\right] \\
& =\lambda\left[l-(b+l) \sum_{m=0}^{x-1} P_{m}(t)\right] .
\end{aligned}
$$

For cost minimization, setting the above expression equal to zero and rearranging terms yields

$$
\sum_{m=0}^{x-1} P_{m}(t)=\frac{l}{b+l}
$$

Second, when $t<g$ follows that

$$
C(x, t)=c_{0}(g-t)+b x P_{0}(t)+b \sum_{n=1}^{x}(x-n) P_{n}(t)+l \sum_{n=x+1}^{\infty}(n-x) P_{n}(t) .
$$

Following similar analysis as above it follows that 


$$
\frac{\partial C}{\partial t}=-c_{0}+\lambda\left[l-(b+l) \sum_{m=0}^{x-1} P_{m}(t)\right]
$$

As before setting this expression equal to zero and rearranging terms yields

$$
\sum_{n=0}^{x-1} P_{n}(t)=\frac{l-\frac{c}{\lambda}}{b+l} .
$$

The second order condition for cost minimization for both cases $t\langle g$ and $t\rangle g$ is

$$
\begin{aligned}
& \frac{\partial^{2} C}{\partial t^{2}}=-\lambda(b+l) \sum_{n=0}^{x-1} P_{n}^{\prime}(t)=\lambda(b+l)\left[-\lambda P_{0}(t)+\lambda \sum_{n=1}^{x-1}\left(P_{n-1}(t)-P_{n}(t)\right)\right] \\
& =\lambda^{2}(b+l)\left[\sum_{n=0}^{x-1} P_{n}(t)-\sum_{n=1}^{x-1} P_{n-1}(t)\right]=\lambda^{2}(b+l)\left[\sum_{n=0}^{x-1} P_{n}(t)-\sum_{m=0}^{x-2} P_{m}(t)\right] \\
& =\lambda^{2}(b+l) P_{x-1}(t)>0 .
\end{aligned}
$$

(b) Using equation (3)

$$
C(x, t)=\operatorname{Max}\left[0, c_{0}(g-t)\right]+x(b+l) \sum_{n=0}^{x} P_{n}(t)-(b+l) \sum_{n=0}^{x} n P_{n}(t)+l(\lambda t-x)
$$

we have that

$$
\begin{aligned}
& C(x+1, t)-C(x, t) \\
& =\operatorname{Max}\left[0, c_{0}(g-t)\right]+(b+l)(x+1) \sum_{n=0}^{x+1} P_{n}(t)-(b+l) \sum_{n=0}^{x+1} n P_{n}(t)+l(\lambda t-x-1) \\
& -\left\{\operatorname{Max}\left[0, c_{0}(g-t)\right]+(b+l) x \sum_{n=0}^{x} P_{n}(t)-(b+l) \sum_{n=0}^{x} n P_{n}(t)+l(\lambda t-x)\right\} \\
& =(b+l) \sum_{n=0}^{x} P_{n}(t)-l .
\end{aligned}
$$

Thus, if $C(x+1, t)-C(x, t)$, then increase inventory by one unit, i.e., increase $x$ by one so long that

$$
C(x+1, t)-C(x, t)=(b+l) \sum_{n=0}^{x} P_{n}(t)-l<0,
$$

which implies that

$$
\sum_{n=0}^{x} P_{n}(t)<\frac{l}{b+l} .
$$

Thus, the cost $C(x, t)$ is minimized for the first $\mathrm{x}$ for which

$$
\sum_{n=0}^{x} P_{n}(t) \geq \frac{l}{b+l}
$$

This implies that, if

$$
P_{0}(t) \geq \frac{l}{b+l},
$$

the cost is minimized at $x=0$. Otherwise, the cost is minimized at that $\mathrm{x}$ for which

$$
\sum_{n=0}^{x-1} P_{n}(t)<\frac{l}{b+l} \leq \sum_{n=0}^{x} P_{n}(t) .
$$

